DIVISION OF THE HUMANITIES AND SOCIAL SCIENCES

CALIFORNIA INSTITUTE OF TECHNOLOGY

PASADENA, CALIFORNIA 91125

PREFERENCE AGGREGATION UNDER UNCERTAINTY: SAVAGE VS. PARETO

Christopher P. Chambers

California Institute of Technology

Takashi Hayashi

University of Rochester

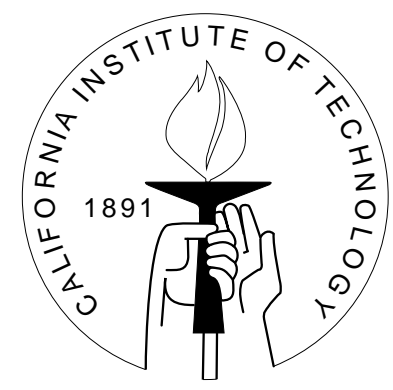

SOCIAL SCIENCE WORKING PAPER 1184

November 2003 


\title{
Preference Aggregation under Uncertainty: Savage vs. Pareto
}

\author{
Christopher P. Chambers and Takashi Hayashi
}

\begin{abstract}
Following Mongin [12, 13], we study social aggregation of subjective expected utility preferences in a Savage framework. We argue that each of Savage's P3 and P4 are incompatible with the strong Pareto property. A representation theorem for social preferences satisfying Pareto indifference and conforming to the state-dependent expected utility model is provided.
\end{abstract}

JEL classification numbers: D71, D81

Key words: Harsanyi's Theorem, preference aggregation, subjective expected utility, Savage's axioms 


\title{
Preference Aggregation under Uncertainty: Savage vs. Pareto
}

\author{
Christopher P. Chambers and Takashi Hayashi *
}

\section{Introduction}

Harsanyi's theorem [8] discusses social aggregation of individual preferences in a risky environment. He shows that when all agents' preferences conform to the axioms of expected utility, if social preference also conforms to the axioms of expected utility and satisfies Pareto indifference with respect to agents' preferences, then social preference can be represented as a weighted sum of agents' expected utility functions. ${ }^{1}$ An example due to Diamond [5] illustrates that Harsanyi's assumptions on social preference may not be compelling.

Formalizing ideas found in Raiffa [15], Mongin [12, 13] pursues a similar program within Savage's [16] framework of subjective uncertainty. If all agents behave according to Savage's axioms, he shows that it is generally impossible for social preference to jointly satisfy Savage's axioms and the strong Pareto property with respect to individual preferences. Two natural responses to this negative conclusion present themselves, both of which were first suggested by Raiffa [15]. One is to condemn the strong Pareto property in a setting of subjective uncertainty. Gilboa, Samet, and Schmeidler [7] discuss this approach, see also Broome [3]. The other approach is to condemn Savage's axioms for social preference. Implicit in most examples which condemn one of these assumptions is the idea that social preference should satisfy the other of the assumptions. In the words of Raiffa, "These issues can be dramatized as a fight between Group Bayesians and Paretians" ([15], p. 234). For a related result, see Hylland and Zeckhauser [9].

Our approach is to understand the basic structure generating the impossibility results, by studying social and individual preferences at an axiomatic level. At an individual

*Division of the Humanities and Social Sciences, Mail Code 228-77, California Institute of Technology, Pasadena, CA 91125. Email: chambers@hss.caltech.edu. Phone: (626) 395-3559 and Department of Economics, University of Rochester, Rochester NY, 14627. Email: taka@troi.cc.rochester.edu. We would like to thank Philippe Mongin and John Weymark for many very helpful comments and suggestions. We would also like to thank Larry Epstein and William Thomson, for many helpful discussions. All errors are our own.

${ }^{1}$ For more on Harsanyi's theorem, see Mongin and D'Aspremont [14] and Weymark [18]. 
level, Savage's axioms are used to deliver a personal notion of probability. The primary axioms in his theory are P2 (sure-thing principle), P3 (eventwise monotonicity), and P4 (weak comparative probability). ${ }^{2}$ In terms of empirical violations of Savage's theory at an individual level, P2 is often criticized. We have no specific criticism against assuming P2 at a social level.

Instead, we discuss P3 and P4. We show by examples that each of these axioms conflicts with the strong Pareto property on its own. Thus, our examples generate impossibility results for a large class of social preferences (for example, social preferences that are probabilistically sophisticated, as defined by Machina and Schmeidler [11]). Informally, each of P3 and P4 carry with them notions of state-independence, which can be understood as the source of the impossibility results.

Our work concludes by studying the most well-known model which generically violates P3 and P4, the state-dependent expected utility model. Arguing that state-independence of social preference is the primary factor driving the impossibility results, Mongin [13] conducts a similar exercise in the Anscombe-Aumann [1] and Karni, Schmeidler, and Vind [10] frameworks. Our theorem gives a counterpart of these results in the Savage framework.

We provide a representation theorem for social preference conforming to the statedependent model and which satisfies Pareto indifference with respect to individual preferences. Under Pareto indifference, social preference can be represented by a weighted sum of individual utility representations. This result is the natural analogue of Harsanyi's theorem [8] in the Savage framework. Gilboa, Samet, and Schmeidler [7] obtain a related result by weakening the Pareto property so that it holds only when all agents in society agree about the likelihood of "relevant" events. Whereas we weaken Savage's axioms as little as possible to obtain a possibility and representation result, they maintain Savage's axioms and weaken the Pareto property as little as possible to obtain a possibility and representation result.

Section 2 describes the model and provides examples and propositions illustrating the conflict between Savage's axioms and the Pareto properties. Section 3 provides a representation theorem in a state-dependent expected utility framework. Finally, Section 4 concludes.

\section{The model and Savage's axioms}

Let $(S, \Sigma)$ be a measurable space, where $S$ is a set of states and $\Sigma$ is a $\sigma$-algebra of events. We use $s$ to denote a generic state and $A$ or $B$ to denote generic events. Let $X$ be a set of outcomes. Define the set of (simple) acts $\mathcal{F}$ as the set of finite-ranged $\Sigma$-measurable mappings $f: S \rightarrow X$. We use $f$ or $g$ to denote a generic act. For $x, y \in X$,

\footnotetext{
${ }^{2}$ The terminology is from Machina and Schmeidler [11].
} 
$A \in \Sigma$, define $x A y \in \mathcal{F}$ as

$$
x A y \equiv\left(\begin{array}{c}
x \text { if } s \in A \\
y \text { if } s \in A^{c}
\end{array}\right) .
$$

With a slight abuse of notation, $x \in X$ also denotes the act whose constant outcome is $x$.

Savage's axioms apply to binary relations $\succeq$ on $\mathcal{F}$. Say that $A \in \Sigma$ is null for $\succeq$ if for all $x \in X$ and all $f \in \mathcal{F}$,

$$
f \sim\left(\begin{array}{c}
x \text { if } s \in A \\
f(s) \text { otherwise }
\end{array}\right) .
$$

For a set of agents $N \equiv\{1, \ldots, n\}$, agent $i$ 's preference is denoted by $\succeq_{i}$ and social preference is denoted by $\succeq_{0}$. Say that $\succeq_{0}$ satisfies Pareto-indifference with respect to $\left(\succeq_{1}, \ldots, \succeq_{n}\right)$ if for all $f, g \in \mathcal{F}$, if for all $i \in N, f \sim_{i} g$, then $f \sim_{0} g$. Say that $\succeq_{0}$ satisfies the weak Pareto property with respect to $\left(\succeq_{1}, \ldots, \succeq_{n}\right)$ if for all $f, g \in \mathcal{F}$, if for all $i \in N, f \succ_{i} g$, then $f \succ_{0} g$. Say that $\succeq_{0}$ satisfies the strong Pareto property with respect to $\left(\succeq_{1}, \ldots, \succeq_{n}\right)$ if it satisfies Pareto indifference with respect to $\left(\succeq_{1}, \ldots, \succeq_{n}\right)$ and if for all $i \in N, f \succeq_{i} g$, with strict preference for some $j \in N$, then $f \succ_{0} g$.

A function $U: \mathcal{F} \rightarrow \mathbb{R}$ is a subjective expected utility functional if there

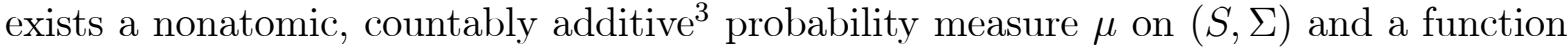
$u: X \rightarrow \mathbb{R}$ such that for all $f \in \mathcal{F}, U(f)=\int_{S} u(f(s)) d \mu(s)$. Say a binary relation $\succeq$ is a subjective expected utility preference if there exists a subjective expected utility functional $U$ such that for all $f, g \in \mathcal{F}$,

$$
f \succeq g \Leftrightarrow U(f) \geq U(g)
$$

For any such $U$ representing a nondegenerate subjective expected utility preference, the associated probability measure $\mu$ is unique, and the function $u$ is unique up to positive affine transformation.

\section{$2.1 \quad$ P3: Eventwise monotonicity}

Savage's axiom P3 states:

P3 For all non-null $A \in \Sigma, x, y \in X, f \in \mathcal{F}$,

$$
x \succeq y \Leftrightarrow\left(\begin{array}{c}
x \text { if } s \in A \\
f(s) \text { if } s \in A^{c}
\end{array}\right) \succeq\left(\begin{array}{c}
y \text { if } s \in A \\
f(s) \text { if } s \in A^{c}
\end{array}\right) .
$$

\footnotetext{
${ }^{3}$ Savage's theorem [16] only delivers a finitely additive probability measure. Arrow [2] discusses conditions which guarantee that the probability measure is countably additive.
} 
A social preference that satisfies P3 cannot generally satisfy the weak Pareto property. The following example illustrates this point.

Example 1 Let $N \equiv\{1,2\}$ and $X \equiv \mathbb{R}_{+}^{2}$. The set $X$ represents distributions of wealth amongst the two agents. Let $\succeq_{0}$ be a social preference over $\mathcal{F}$. Assuming each agent likes more wealth to less and cares only about her own wealth, a plausible social ranking is $(0,100) \sim_{0}(100,0)$. Let $A, A^{c} \in \Sigma$. Suppose agent one believes $A$ is more likely than $A^{c}$ and agent two believes $A^{c}$ is more likely than $A$. Then for $i=1,2$,

$$
(100,0) A(0,100) \succ_{i}(0,100) A(100,0) .
$$

By the weak Pareto property,

$$
(100,0) A(0,100) \succ_{0}(0,100) A(100,0) .
$$

Here, $\succeq_{0}$ violates P3. To see this, suppose $\succeq_{0}$ satisfies P3. As $(100,0) \sim_{0}(0,100)$, and as $\succeq_{0}$ satisfies P3,

$$
(100,0) \sim_{0}(100,0) A(0,100)
$$

and

$$
(0,100) A(100,0) \sim_{0}(0,100) .
$$

By transitivity, the ranking $(100,0) \succ_{0}(0,100)$ holds, a contradiction.

\section{$2.2 \quad$ P4: Weak comparative probability}

Savage's axiom P4 states:

$\mathbf{P} 4$ For all $\bar{x}, \underline{x}, \bar{y}, \underline{y}$ such that $\bar{x} \succ \underline{x}$ and $\bar{y} \succ \underline{y}$, for all $A, B \in \Sigma$

$$
\bar{x} A \underline{x} \succ \bar{x} B \underline{x} \Leftrightarrow \bar{y} A \underline{y} \succ \bar{y} B \underline{y} .
$$

Example 2 shows that P4 is also incompatible with the strong Pareto property.

Example 2 Let $N \equiv\{1,2\}$ and $X \equiv R_{+}^{2}$, where the numerical quantities again represent monetary values. Each agent only cares about the amount she receives. Suppose again that agent one believes $A$ is more likely than $A^{c}$, and agent two believes $A^{c}$ is more likely than $A$. By strong Pareto, $(100,0) \succ_{0}(0,0)$ and $(0,100) \succ_{0}(0,0)$. Thus,

$$
(100,0) A(0,0) \succ_{1}(0,0) A(100,0)
$$

and

$$
(100,0) A(0,0) \sim_{2}(0,0) A(100,0) .
$$

By the strong Pareto property,

$$
(100,0) A(0,0) \succ_{0}(0,0) A(100,0) .
$$


By a symmetric argument,

$$
(0,100) A(0,0) \prec_{0}(0,0) A(0,100) .
$$

These rankings clearly violate $\mathrm{P} 4$. The structure of this example is closely related to an example found in Gilboa, Samet, and Schmeidler [7]. It formally illustrates the reasoning which may lead one to reject the Pareto principle.

\subsection{General incompatibility results}

The following propositions are general versions of the examples.

The first proposition follows directly from Example 1. For any preference relation $\succeq$ satisfying P4, a likelihood relation $\succeq^{l}$ on $\Sigma$ can be defined as follows: $A \succeq^{l} B$ if and only if there exist $x, x^{\prime} \in X$ such that $x \succ x^{\prime}$ and $x A x^{\prime} \succeq x B x^{\prime} .^{4}$

Proposition 1: Suppose $\succeq_{1}$ and $\succeq_{2}$ satisfy P4 and for all $i=1,2, A \succeq_{i}^{l} B \Leftrightarrow B^{c} \succeq_{i}^{l} A^{c}$. Suppose there exist $\bar{x}, \underline{x} \in X$ such that $\bar{x} \succ_{1} \underline{x}, \underline{x} \succ_{2} \bar{x}$, and $\bar{x} \sim_{0} \underline{x}$. If $\succeq_{0}$ satisfies P3 and the strong Pareto property with respect to $\left(\succeq_{1}, \succeq_{2}\right)$, then $\succeq_{1}^{l}=\succeq_{2}^{l}$.

The next proposition illustrates a related point. If individuals' "beliefs" are different, then aggregation under the strong Pareto property and P3 is possible only if their "tastes" are the same.

Proposition 2: Suppose $\succeq_{1}$ and $\succeq_{2}$ satisfy P3 and that there exist $\bar{x}, \underline{x}, \bar{y}, \underline{y}$ such that $\bar{x} \succ_{1} \underline{x}$ and $\bar{y} \succ_{2} \underline{y}$. Suppose there exist $A \in \Sigma$ such that $A$ is non-null for $\succeq_{1}$ and null for $\succeq_{2}$, and $B \in \Sigma$ such that $B$ is non-null for $\succeq_{2}$ and null for $\succeq_{1}$. If $\succeq_{0}$ satisfies P3 and satisfies the strong Pareto property with respect to $\left(\succeq_{1}, \succeq_{2}\right)$, then $\left.\succeq_{1}\right|_{X}=\left.\succeq_{2}\right|_{X}$

Proof: We first show that each of $A$ and $B$ is non-null for $\succeq_{0}$. As $\succeq_{1}$ satisfies P3 and $A$ is non-null for $\succeq_{1}, \bar{x} A \underline{x} \succ_{1} \underline{x}$. As $A$ is null for $\succeq_{2}, \bar{x} A \underline{x} \sim_{2} \underline{x}$. By the strong Pareto property, $\bar{x} A \underline{x} \succ_{0} \underline{x}$, so that $A$ is non-null for $\succeq_{0}$. The proof for $B$ is symmetric.

Suppose the statement of the proposition is false. Thus, $\left.\succeq_{1}\right|_{X} \neq\left.\succeq_{2}\right|_{X}$, and without loss of generality, there exist $x, y \in X$ such that $x \succeq_{1} y$ and $y \succ_{2} x$. As $\succeq_{1}$ satisfies P3, $x A y \succeq_{1} y$, and as $A$ is null for $\succeq_{2}, x A y \sim_{2} y$. By the strong Pareto property, $x A y \succeq_{0} y$. As $A$ is non-null for $\succeq_{0}$, by P $3, x \succeq_{0} y$.

As $\succeq_{2}$ satisfies P3 and as $B$ is non-null for $\succeq_{2}, y \succ_{2} x B y$, and as $B$ is null for agent $\succeq_{1}, y \sim_{1} x B y$. By the strong Pareto property, $y \succ_{0} x B y$. As $B$ is non-null for $\succeq_{0}$, by P3, $y \succ_{0} x$. But we previously concluded that $x \succeq_{0} y$, a contradiction.

The last proposition follows directly from Example 2 .

\footnotetext{
${ }^{4}$ Propositions 1, 2, and 3 are stated for environments with only two agents. Similar results can be established for any number of agents, by partitioning the set of agents into two types, where each type has a preference relation corresponding to one of the two preference relations in the propositions.
} 
Proposition 3: Suppose $\succeq_{1}$ and $\succeq_{2}$ satisfy P4 and for all $i=1,2$, for all $x, x^{\prime} \in X$, $A, B \in \Sigma$,

$$
x \sim_{i} x^{\prime} \Longrightarrow x A x^{\prime} \sim_{i} x B x^{\prime} .
$$

Suppose there exist $\bar{x}, \underline{x}, \bar{y}, \underline{y} \in X$ such that $\bar{x} \succ_{1} \underline{x}, \bar{x} \sim_{2} \underline{x}, \bar{y} \sim_{1} \underline{y}, \bar{y} \succ_{2} \underline{y} . \quad$ If $\succeq_{0}$ satisfies P4 and the strong Pareto property with respect to $\left(\succeq_{1}, \succeq_{2}\right)$, then $\succeq_{1}^{l}=\succeq_{2}^{l} .{ }^{5}$

\section{A possibility result}

Each of $\mathrm{P} 3$ and $\mathrm{P} 4$ can be thought of as requirements of state-independence. Thus, in this section, we show that if social preference is not required to satisfy P3 and P4, Paretian aggregation is possible under the state-dependent expected utility model. To this end, say $U: \mathcal{F} \rightarrow \mathbb{R}$ is a state-dependent subjective expected utility functional if there exists a nonatomic, countably additive probability measure $\mu$ on $(S, \Sigma)$ and a function $u: X \times S \rightarrow \mathbb{R}$ such that for all $x \in X, u(x, \cdot): S \rightarrow \mathbb{R}$ is $\Sigma$-measurable and $\mu$-integrable and for all $f \in \mathcal{F}, U(f)=\int_{S} u(f(s), s) d \mu(s)$. Say a binary relation $\succeq$ is a statedependent subjective expected utility preference if there exists a state-dependent subjective expected utility functional $U$ such that for all $f, g \in \mathcal{F}$,

$$
f \succeq g \Leftrightarrow U(f) \geq U(g) .
$$

For more information about state-dependent subjective expected utility preferences, see Wakker and Zank [17].

The probability measure component of a state-dependent subjective expected utility functional is not unique. Hence, we cannot refer to society's "beliefs" in an unambiguous way. This is an artifact of the incompatibility of $\mathrm{P} 4$ with Paretian aggregation.

Theorem 1: Suppose that for all $i \in N, \succeq_{i}$ is a subjective expected utility preference represented by $U_{i}: \mathcal{F} \rightarrow \mathbb{R}$. Then $\succeq_{0}$ is a state-dependent subjective expected utility preference which satisfies Pareto-indifference with respect to $\left(\succeq_{1}, \ldots, \succeq_{n}\right)$ if and only if there exists a vector $\lambda \in \mathbb{R}^{N}$ such that for all $f, g \in \mathcal{F}$,

$$
f \succeq_{0} g \Leftrightarrow \sum_{N} \lambda_{i} U_{i}(f) \geq \sum_{N} \lambda_{i} U_{i}(g) .
$$

Representations corresponding to stronger Pareto properties can similarly be derived using Lemma 1 of Appendix A and the general representation theorems of DeMeyer and Mongin [4]. Mongin [13] proves a related theorem using the added structure of the Anscombe-Aumann [1] framework. He also showed in [12] that if the probability

\footnotetext{
${ }^{5}$ We could replace the hypothesis $\bar{x} \succ_{1} \underline{x}, \bar{x} \sim_{2} \underline{x}, \bar{y} \sim_{1} \underline{y}, \bar{y} \succ_{2} \underline{y}$ with the hypothesis that $\bar{x} \succ_{1}$ $\underline{x}, \bar{x} \preceq_{2} \underline{x}, \bar{x} \succ_{0} \underline{x}, \bar{y} \preceq_{1} \underline{y}, \bar{y} \succ_{2} \underline{y}, \bar{y} \succ_{0} \underline{y}$. The first of these hypotheses derives the social preference in the second hypothesis from the strong Pareto property.
} 
measures of all agents and society can have atoms, then Theorem 1 does not hold in a Savage setup. Theorem 1 is closely related to Harsanyi's original result [8], in that it is driven by the sure-thing principle (which is closely tied to the independence axiom of expected utility theory which drives Harsanyi's result). In fact, Harsanyi's Theorem should be viewed as a special case of our Theorem 1, where social preference satisfies Savage's axioms and the likelihood relations of all agents and of society coincide.

The proof of Theorem 1 is in the Appendix. In order to prove Theorem 1, we establish in Lemma 1 that the utility possibilities set for a collection of state-dependent expected utility maximizers is convex, a fact which comes for free in the AnscombeAumann model. After establishing this, we apply a general representation theorem of DeMeyer and Mongin [4].

\section{Conclusion}

Mongin's [12] negative results make clear that Paretian aggregation is incompatible with the expected utility model for social preference. Going further, he also suggests the idea that the problem with Paretian aggregation is the state-dependence of preferences satisfying Savage's axioms [13]. Our first contribution is to work at a primitive level, identifying exactly which of Savage's axioms are the source of the negative results. We determine that two of Savage's axioms are incompatible with Paretian aggregation-P3 and $\mathrm{P} 4$. Our second contribution is to demonstrate the possibility of Paretian aggregation without P3 and P4 by providing a representation for social preference conforming to the state-dependent expected utility model.

\section{Appendix-Proof of Theorem 1}

Lemma 1 establishes that the utility possibility set for a collection of state-dependent expected utility maximizers is convex. This result relies on a generalization of Lyapunov's Convexity Theorem, due to Dvoretzky, Wald, and Wolfovitz [6].

Lemma 1: Suppose $\left(U_{1}, \ldots, U_{m}\right)$ is a vector of state-dependent expected utility functionals. Then

$$
\left\{\left(U_{1}(f), \ldots, U_{m}(f)\right): f \in \mathcal{F}\right\}
$$

is convex.

Proof: We need to show that for all $f, g \in \mathcal{F}, \alpha \in[0,1]$, there exists some $h \in \mathcal{F}$ such that for all $i=1, \ldots, m, U_{i}(h)=\alpha U_{i}(f)+(1-\alpha) U_{i}(g)$.

Therefore, let $f^{*}, g^{*} \in \mathcal{F}, \alpha^{*} \in[0,1]$. 
Let $X\left(f^{*}, g^{*}\right) \equiv\left(\operatorname{range}\left(f^{*}\right)\right) \cup\left(\right.$ range $\left.\left(g^{*}\right)\right)$. As $f^{*}, g^{*}$ are simple acts, $\left|X\left(f^{*}, g^{*}\right)\right|<$ $\infty$. Let $\mathcal{F}\left(f^{*}, g^{*}\right) \subset \mathcal{F}$ be the set of acts whose range lies in $X\left(f^{*}, g^{*}\right)$.

By assumption, for all $i=1, \ldots, m$, there exists $u_{i}: X \times S \rightarrow \mathbb{R}$ and $\mu_{i}$ on $(S, \Sigma)$ such that $U_{i}(f) \equiv \int_{S} u_{i}(f(s), s) d \mu_{i}(s)$. For all $i=1, \ldots, m$, and for all $x \in X\left(f^{*}, g^{*}\right)$, let $\nu_{i}^{x}$ be a measure on $\Sigma$ defined by

$$
\nu_{i}^{x}(E) \equiv \int_{E} u_{i}(x, s) d \mu_{i}(s)
$$

Then for all $i \in N, x \in X\left(f^{*}, g^{*}\right), \nu_{i}^{x}$ is countably additive, nonatomic, and finite. ${ }^{6}$ By definition of the integral, for all $i \in N, f \in \mathcal{F}\left(f^{*}, g^{*}\right)$

$$
U_{i}(f)=\sum_{x \in X\left(f^{*}, g^{*}\right)} \nu_{i}^{x}\left(f^{-1}(x)\right)
$$

Let $\Pi$ be the set of $\Sigma$-measurable ordered $X\left(f^{*}, g^{*}\right)$-partitions. Formally, $\Pi$ is defined as the set of functions $P: X\left(f^{*}, g^{*}\right) \rightarrow \Sigma$ satisfying $\left.i\right) \bigcup_{X\left(f^{*}, g^{*}\right)} P(x)=S$ and $\left.i i\right)$ for all $x, y \in X\left(f^{*}, g^{*}\right)$ such that $x \neq y, P(x) \cap P(y)=\emptyset$.

Clearly, there is a bijection $\psi$ between $\mathcal{F}\left(f^{*}, g^{*}\right)$ and $\Pi$, given by for all $f \in \mathcal{F}\left(f^{*}, g^{*}\right)$ and for all $x \in X\left(f^{*}, g^{*}\right), \psi(f)(x) \equiv f^{-1}(x)$. In particular,

$$
A \equiv\left\{\left(\nu_{i}^{x}(P(x))\right)_{i, x} \subset \mathbb{R}^{m \times X\left(f^{*}, g^{*}\right)}: P \in \Pi\right\}
$$

is equal to

$$
B \equiv\left\{\left(\nu_{i}^{x}\left(f^{-1}(x)\right)\right)_{i, x} \subset \mathbb{R}^{m \times X\left(f^{*}, g^{*}\right)}: f \in \mathcal{F}\left(f^{*}, g^{*}\right)\right\} .
$$

By Theorems 1 and 4 of Dvoretzky, Wald, and Wolfowitz [6], it follows that $A$ is convex. ${ }^{7}$ Thus $B$ is also convex.

By summing the columns of the elements of $B$, we obtain $\left\{\left(U_{1}(f), \ldots, U_{m}(f)\right): f \in \mathcal{F}\left(f^{*}, g^{*}\right)\right\}$. Convexity is preserved under this summation. Lastly, note that $\left\{\left(U_{i}\left(f^{*}\right)\right)_{i},\left(U_{i}\left(g^{*}\right)\right)_{i}\right\} \subset\left\{\left(U_{1}(f), \ldots, U_{m}(f)\right): f \in \mathcal{F}\left(f^{*}, g^{*}\right)\right\}$. Thus, there exists some $h \in \mathcal{F}\left(f^{*}, g^{*}\right)$ such that for all $i=1, \ldots, m$, $U_{i}(h)=\alpha^{*} U_{i}\left(f^{*}\right)+\left(1-\alpha^{*}\right) U_{i}\left(g^{*}\right)$.

\footnotetext{
${ }^{6}$ To see that $\nu_{i}^{x}$ is nonatomic, suppose that it is not. Then there exists some $E \in \Sigma$ such that $\nu_{i}^{x}(E)>0$, and for all $F \subset E, \nu_{i}^{x}(F) \in\left\{0, \nu_{i}^{x}(E)\right\}$. Let $\left\{E_{m}\right\}_{m=1}^{\infty}$ be a sequence such that $E_{1}=E$, and for all $m, E_{m} \subset E_{m-1}, \mu_{i}\left(E_{m}\right)=\frac{1}{2} \mu_{i}\left(E_{m-1}\right)$, and $\nu_{i}^{x}\left(E_{m}\right)=\nu_{i}^{x}(E)$. By countable additivity, $\mu_{i}\left(\bigcap_{m=1}^{\infty} E_{m}\right)=0$ and $\nu_{i}^{x}\left(\bigcap_{m=1}^{\infty} E_{m}\right)=\nu_{i}^{x}(E)>0$, a contradiction. Finiteness follows as $u_{i}(x, \cdot)$ is $\mu_{i}$-integrable.

${ }^{7}$ This follows as Theorems 1 and 4 of Dvoretzky, Wald, and Wolfowitz imply that $\left\{\left(\nu_{i}^{x}(P(y))\right)_{(i, x), y} \subset \mathbb{R}^{\left(m \times X\left(f^{*}, g^{*}\right)\right) \times X\left(f^{*}, g^{*}\right)}: P \in \Pi\right\}$ is convex. The set $A$ is a projection of this set on the subspace in which $x=y$.
} 
We now prove the theorem.

Proof: It is obvious that if there exists a vector $\lambda \in \mathbb{R}^{N}$ such that for all $f, g \in \mathcal{F}$, $f \succeq_{0} g \Leftrightarrow \sum_{N} \lambda_{i} U_{i}(f) \geq \sum_{N} \lambda_{i} U_{i}(g)$, then $\succeq_{0}$ satisfies Pareto indifference with respect to $\left(\succeq_{1}, \ldots, \succeq_{n}\right)$. We will now show that it is a state-dependent subjective expected utility preference. If for all $f, g \in \mathcal{F}, f \sim_{0} g$, then the claim is obvious. So, assume there exist $f^{\prime}, g^{\prime} \in \mathcal{F}$ such that $f^{\prime} \succ_{0} g^{\prime}$.

For all $i \in N, U_{i}$ has two components, a utility index $u_{i}$, and a probability measure $\mu_{i}$. Let $\mu \equiv \frac{\sum_{N} \mu_{i}}{|N|}$. Then $\mu$ is a probability measure defined on $(S, \Sigma)$. For all $i \in N$, $\mu_{i}$ is absolutely continuous with respect to $\mu$. By the Radon-Nikodym Theorem, for all $i \in N$, there exists a $\Sigma$-measurable, $\mu$-integrable function $h_{i}: S \rightarrow \mathbb{R}$ such that for all $f \in \mathcal{F}$

$$
\int_{S} u_{i}(f(s)) d \mu_{i}(s)=\int_{S} u_{i}(f(s)) h_{i}(s) d \mu(s) .
$$

Thus, for all $f \in \mathcal{F}$,

$$
\sum_{N} \lambda_{i} U_{i}(f)=\int_{S}\left(\sum_{N} \lambda_{i} u_{i}(f(s)) h_{i}(s)\right) d \mu(s) .
$$

Let $u: X \times S \rightarrow \mathbb{R}$ be defined by

$$
u(x, s) \equiv \sum_{N} \lambda_{i} u_{i}(x) h_{i}(s)
$$

Thus, for all $x \in X, u(x, \cdot)$ is $\Sigma$-measurable and $\mu$-integrable, and for all $f \in \mathcal{F}$,

$$
\sum_{N} \lambda_{i} U_{i}(f)=\int_{S} u(f(s), s) d \mu(s) .
$$

Conversely, suppose that for all $i \in N, \succeq_{i}$ is a subjective expected utility preference. Suppose that $\succeq_{0}$ is a state-dependent subjective expected utility preference which satisfies Pareto indifference with respect to $\left(\succeq_{1}, \ldots, \succeq_{n}\right)$.

For all $i \in N$, let $U_{i}: \mathcal{F} \rightarrow \mathbb{R}$ be a subjective expected utility functional representing $\succeq_{i}$, and $U_{0}$ a state-dependent subjective expected utility functional representing $\succeq_{0}$. By Lemma 1 above and Proposition 1 of DeMeyer and Mongin [4], there exist $\lambda \in \mathbb{R}^{N}$ and $K \in \mathbb{R}$ such that for all $f \in \mathcal{F}$,

$$
U_{0}(f)=K+\sum_{N} \lambda_{i} U_{i}(f) .
$$

Thus, for all $f, g \in \mathcal{F}, f \succeq_{0} g$ if and only if $K+\sum_{N} \lambda_{i} U_{i}(f) \geq K+\sum_{N} \lambda_{i} U_{i}(g)$. Equivalently, $f \succeq_{0} g$ if and only if $\sum_{N} \lambda_{i} U_{i}(f) \geq \sum_{N} \lambda_{i} U_{i}(g)$. 


\section{References}

[1] F.J. Anscombe and R.J. Aumann, A definition of subjective probability, Annals of Mathematical Statistics 34 (1963), 199-205.

[2] K. Arrow, "Essays in the Theory of Risk-Bearing," North-Holland, Amsterdam, 1970.

[3] J. Broome, "Weighing Goods," Basil Blackwood, Cambridge MA, 1991.

[4] B. DeMeyer and P. Mongin, A note on affine aggregation, Economics Letters 47 (1995), 177-183.

[5] P. A. Diamond, Cardinal welfare, individualistic ethics, and interpersonal comparisons of utility: a comment, Journal of Politicial Economy 75 (1967), 765-766.

[6] A. Dvoretzky, A. Wald, and J. Wolfovitz, Relations among certain ranges of vector measures, Pacific Journal of Mathematics 1 (1951), 59-74.

[7] I. Gilboa, D. Samet, and D. Schmeidler, "Utilitarian aggregation of beliefs and tastes," manuscript, 2001.

[8] J.C. Harsanyi, Cardinal welfare, individualistic ethics, and interpersonal comparisons of utility, Journal of Political Economy 63 (1955), 309-321.

[9] A. Hylland and R. Zeckhauser, The impossibility of Bayesian group decision making with separate aggregation of beliefs and values, Econometrica 47 (1979), 1321-1336.

[10] E. Karni, D. Schmeidler, and K. Vind, On state dependent preferences and subjective probabilities, Econometrica 51 (1983), 1021-1031.

[11] M. Machina and D. Schmeidler, A more robust definition of subjective probability, Econometrica 60 (1992), 745-780.

[12] P. Mongin, Consistent Bayesian aggregation, Journal of Economic Theory 66 (1995), 313-351.

[13] P. Mongin, The paradox of the Bayesian experts and state-dependent utility theory, Journal of Mathematical Economics 29 (1998), 331-361.

[14] P. Mongin and C. D'Aspremont, Utility theory and ethics, in "Handbook of Utility Theory, Volume 1, Principles," (S. Barbera, P. J. Hammond, and C. Seidl, Eds.), Kluwer Academic Publishers, London.

[15] H. Raiffa, "Decision Analysis," Addison-Wesley, Reading, MA, 1968.

[16] L.J. Savage, "The Foundations of Statistics," John Riley, New York, 1954.

[17] P.P. Wakker and H. Zank, State dependent expected utility for Savage's state space, Mathematics of Operations Research 24 (1999), 8-34. 
[18] J. Weymark, A reconsideration of the Harsanyi-Sen debate on utilitarianism, in "Interpersonal Comparisons of Well-Being," (J. Elster and J.E. Roemer, Eds.) Cambridge University Press, Cambridge, 1991. 